\title{
Effect of baffle shape in heat transfer for jet impingement on a solid block
}

\author{
Muthukannan Marimuthu ${ }^{1}$, Uthayakumar $^{2}$, Rajesh Kanna $\mathrm{P}^{3}$, Paweł Ocłon, ${ }^{* 4}$, Jan Taler ${ }^{5}$, and Dawid Taler ${ }^{6}$ \\ ${ }^{1}$ Department of Mechanical Engineering, Kalasalingam University, Anand Nagar,Krishnan Koil,Tamilnadu,India 626126 \\ ${ }^{2}$ Department of Mechanical Engineering, Kalasalingam University, Anand Nagar,Krishnan Koil,Tamilnadu,India 626126 \\ ${ }^{3}$ Department of Mechanical Engineering, College of Engineering and Computing, Alghurair University,, Dubai. UAE. \\ 4,5,6 Institute of Thermal Power Engineering, Cracow University of Technology, Kraków, Poland.
}

\begin{abstract}
The numerical solution solution is obtained for fluid flow and heat transfer in a confined impinging slot on a solid block with the presence of baffles. In order to consider the effect of baffle shape the rectangular and semi circular baffles are considered and for the effect for Reynolds number the Reynolds number is varied from 100 to 300 with the step of 50. The present study reveals the vital impact of Baffle shape and Reynolds number (Re) on the fluid flow and heat transfer characteristics over a wide range. It is finally added that the presence of baffle improves the Nusselt number. The Nusselt number increases with the increase of Reynolds number. The present study proved that, the primary peak of Nusselt number occurs nearer to the reattachment length. The secondary peak of Nusselt number occurs nearer to the baffle. It is observed that for semi circle baffle the velocity attains maximum one compared to rectangular baffle.
\end{abstract}

\section{Introduction}

The jet impingement cooling technique is used in industries like turbine blade cooling, rocket launcher cooling, electrical, metal annealing, and textile drying and electronic equipments in order to confiscate a huge amount of heat. The common types of impingement are confined and unconfined jets. The design and fabrication of confined jets are very complicated compared to unconfined jets. There are many numerical and experimental investigations are conducted to find out the effect of confinement wall on jet impingement. The experimental and numerical predictions are mostly related to turbulent jet impingement to have fast cooling. But in the case of cooling of electronic equipments the area need to be cooled is small and also the velocity. In order to consider the present situation, the laminar regime is considered for the entire computational domain.

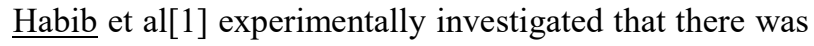
increase in average nusselt number with the increase of Reynolds number and block height. Hussein M.Maghrabie et al [2] numerically investigated the heat transfer and pressure drop of in line array of heated obstacles cooled by jet impingement in cross flow. They concluded that there was a significant change in jet impingent cross flow and normal flow of jet impingement. WithadaJedsadaratanachai etal [3] predicted numerically $\mathrm{V}$ baffles can induce a drastic increase in Nusselt number and higher friction.
Yang etal [4] investigated experimental as well as numerically in array of jet impingement on a concave surfaces.The finally concluded that,increase in jet diameter hole will not improve the heat transfer significantly. Eiamsa-ard[5] et al numerically examined the fluid flo and heat transfer chararteristics in the presence of triangular wavy baffles. They concluded that for $30^{\circ}$ and $45^{\circ}$ baffles endow with more nusselt number compared to $60^{\circ}$ baffles. Alsanossi M.Aboghrara [6] examined analysis of solar air heater with jet impingement on corrugated absorber plate. They exposed that corrugated plate is the strong function of heat transfer enhancement. In previous series of studies performed by author [7\&8]concentrated more on fluid flow structure behavior of different fluids like air, water and nano fluid jet impingement on a solid block. In the present paper the author revealed a numerical study report on the effect of imposing the different shape of baffles on jet impingement on a solid block. The primary aim of this study is to elucidate the enhancement in heat transfer by incorporating baffles by (i.e. increasing area).

\section{Problem Descriptions}

A The schematic diagram for single slot jet impingement on a solid block in the presence of baffles are shown in figure 1. The air jet come out from a nozzle of width $\mathrm{W}$, with a uniform velocity (U), impinges normal to a

\footnotetext{
* Corresponding author: poclon@mech.pk.edu.pl
} 
computational domain which is called impingement plate at a distance of $\mathrm{L}_{\mathrm{y}}$ from the nozzle. The horizontal and vertical velocity are considered as $\mathrm{U}$ and $\mathrm{V}$ are respectively. Since the computational domain is symmetry about vertical or y-axis, it is prudent to model and simulate only right hand of the computational domain thereby saving precious computing power and time.

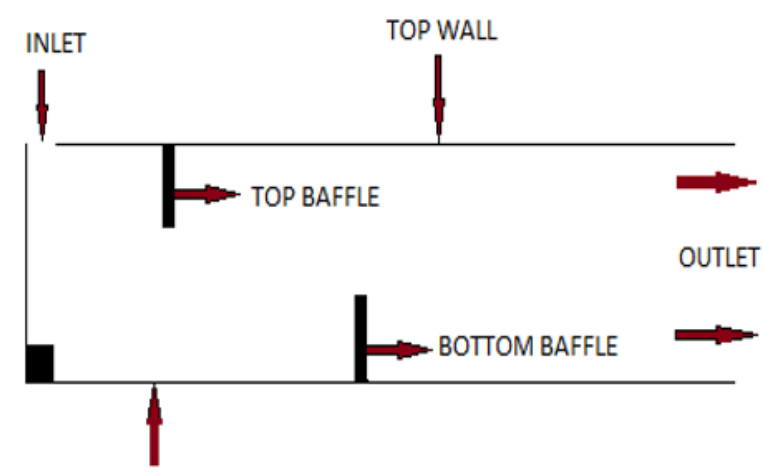

\section{BOTTOM WALL}

(HOT WALL)

Fig. 1(a).Schematic diagram of computational domain with rectangular deflector

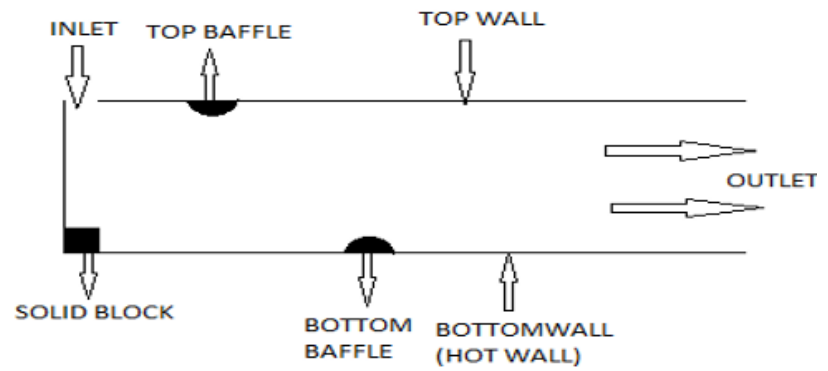

Fig. 1(b). Schematic diagram of computational domain with baffle shaped deflector

The inlet velocity of air (U) is calculated by using the formula: $\operatorname{Re}=\left(\rho \mathrm{UD}_{\mathrm{h}} / \mu\right)$ where $\rho$ denotes the density of the fluid; $D_{h}$ the hydraulic diameter, and $\mu$-dynamic viscosity of the fluid. The deflectors are provided at the top and bottom walls at equal distance having same area. Dimensionless velocity $(\mathrm{U} \& \mathrm{~V})$ are determined by $\mathrm{U}=\mathrm{u}$ $/ \mathrm{U}_{\text {in }}, \mathrm{V}=\mathrm{v} / \mathrm{U}_{\text {in }}$ where $\mathrm{u}$ and $\mathrm{v}$ are the dimensional velocities and $U_{\text {in }}$ is considered as the reference velocity and equal to unity.The aspect ratio (AR) is defined as length of the domain in $\mathrm{Y}$ direction $\left(\mathrm{L}_{\mathrm{y}}\right)$ to hydraulic diameter $\left(D_{h}\right)$

\subsection{Boundary Conditions}

The numerical study had a uniform wall temperature at the bottom wall and top wall is considered as an adiabatic one. Uniform velocity is considered as inlet and pressure outflow is considered at outlet. Symmetry condition is adopted by considering right hand of the computational domain inorder to reduce the computational time.

\subsection{Assumptions}

The following assumptions were made while solving this problem:

- Flow was taken as laminar i.e. Reynolds number varies from 50 to 300 with the step of $\mathrm{Re}=50$ only since the presence of the block induces turbulence at low Reynolds number also.

- Incompressible fluid.

- The case solved here is that of non-conjugate heat transfer i.e. the effect of the conductivity of the slab material is not taken into account.

- The geometry at the outlet is assumed to be so long that the flow eventually gets fully developed.

\subsection{Commercial Package Used}

The continuity equation, momentum equation and energy equation are solved by commercial package ANSYSCFD15.0.

$\frac{\partial \mathrm{U}}{\partial \mathrm{X}}+\frac{\partial \mathrm{V}}{\partial \mathrm{Y}}=0 \quad$ Continuity Equation

$\mathrm{X}$ Momentum Equation

$$
\mathrm{U}\left(\frac{\partial \mathrm{U}}{\partial \mathrm{X}}\right)+\mathrm{V} \frac{\partial \mathrm{U}}{\partial \mathrm{Y}}=-\frac{\partial \mathrm{P}}{\partial \mathrm{X}}+\frac{1}{\operatorname{Re}}\left(\frac{\partial^{2} \mathrm{U}}{\partial \mathrm{X}^{2}}+\frac{\partial^{2} \mathrm{U}}{\partial \mathrm{Y}^{2}}\right)
$$

Y Momentum Equation

$\mathrm{U} \frac{\partial \mathrm{V}}{\partial \mathrm{X}}+\mathrm{V} \frac{\partial \mathrm{V}}{\partial \mathrm{Y}}=-\frac{\partial \mathrm{P}}{\partial \mathrm{Y}}+\frac{1}{\mathrm{Re}}\left(\frac{\partial^{2} \mathrm{~V}}{\partial \mathrm{X}^{2}}+\frac{\partial^{2} \mathrm{~V}}{\partial \mathrm{Y}^{2}}\right)$

Energy equation:

$\mathrm{U} \frac{\partial \theta}{\partial \mathrm{X}}+\mathrm{V} \frac{\partial \theta}{\partial \mathrm{Y}}=\frac{1}{\operatorname{RePr}}\left(\frac{\partial^{2} \theta}{\partial \mathrm{X}^{2}}+\frac{\partial^{2} \theta}{\partial \mathrm{Y}^{2}}\right)$

The non-dimensional variables considered for this problem are,

$\mathrm{X}=\mathrm{Lx} / \mathrm{D}_{\mathrm{h}}, \mathrm{Y}=\mathrm{Ly} / \mathrm{D}_{\mathrm{h}}, \mathrm{U}=\mathrm{u} / \mathrm{U}_{\text {in }}, \mathrm{V}=\mathrm{v} / \mathrm{U}_{\text {in }} \mathrm{P}=\mathrm{p} / \rho$ $\mathrm{U}_{\text {in }}^{2}$

$\operatorname{Re}=\left(\rho \mathrm{D}_{\mathrm{h}} / \mu\right)$ and $\mathrm{Nu}=\left(-\frac{\partial \theta}{\partial \mathrm{Y}}\right)_{\mathrm{y}=0}$

where $\theta_{\mathrm{f}}=\frac{\mathrm{T}_{\mathrm{f}}-\mathrm{T}_{\mathrm{c}}}{\mathrm{T}_{\mathrm{h}}-\mathrm{T}_{\mathrm{c}}}$ is the non dimensional temperature in the fluid region and

$\theta_{\mathrm{s}}=\frac{\mathrm{T}_{\mathrm{w}}-\mathrm{T}_{\mathrm{c}}}{\mathrm{T}_{\mathrm{h}}-\mathrm{T}_{\mathrm{c}}}$ is the non dimensional temperature in the solid region.

The term $\theta_{\mathrm{s}}$ is always 1 for the non-conjugate case, i.e., the effect of the slab thickness is neglected. Here the temperature of the wall is assumed as the temperature of the hot fluid $\left(\mathrm{T}_{\mathrm{w}}=\mathrm{T}_{\mathrm{h}}\right)$.

\subsection{Validation of the problem}

In order to prove our algorithm the validation work has been done against sivasamy et al paper[4]. The centers of the primary and secondary vortexes for aspect ratio 5 for Reynolds number from 100 to 400, the same for Reynolds number 200 and aspect ratio 2 to 5 has been used as the parameters for validation. 


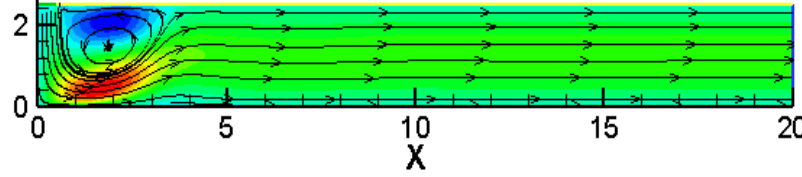

Fig. 2. X-velocity contours and streamlines for aspect ratio 5 and $\operatorname{Re}=100$

Table 1 compares the center of primary vortex in $\mathrm{x}$ coordinate produced by present algorithm with sivasamy et al result. Table 1 compares the center of primary vortex in y coordinate produced by present algorithm with sivasamy et al result.

Table 1 Centres for primary vortex in $\mathrm{X} \& \mathrm{Y}$-coordinate.

\begin{tabular}{|c|l|l|l|l|}
\hline \multirow{2}{*}{$\operatorname{Re}$} & \multicolumn{4}{|c|}{ Primary Vortex } \\
\cline { 2 - 5 } & \multicolumn{4}{|c|}{ Coordinates } \\
\cline { 2 - 5 } & Present & $\begin{array}{c}\text { Sivasamy } \\
\text { et al }\end{array}$ & Present & $\begin{array}{c}\text { Sivasamy } \\
\text { et al }\end{array}$ \\
\hline 100 & 1.90 & 1.90 & 1.43 & 1.41 \\
\hline 200 & 3.12 & 3.07 & 1.52 & 1.49 \\
\hline 300 & 4.08 & 4.05 & 1.60 & 1.56 \\
\hline 400 & 4.86 & 4.84 & 1.64 & 1.63 \\
\hline 500 & 5.43 & 5.42 & 1.71 & 1.67 \\
\hline
\end{tabular}

The figure 3 shows that,the coefficient of friction in bottom wall is compared with sivasamy et al and it is having good agreement also.

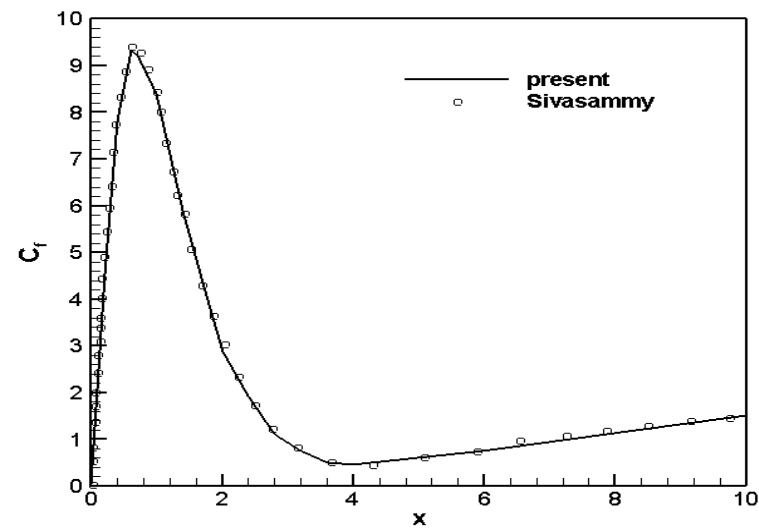

Fig 3. Comparison of Bottom wall friction (Cf) with sivasamy et al

The coefficient of friction in bottom wall is compared with sivasamy et al and it is having good agreement also.

For validation of flow field investigation, the extraction of vortex centre and coefficient of friction is extracted along the computational domain and it is compared with sivasamy et al and it is having good agreement also.

For validation of heat transfer result, the nusselt number calculated along the computational domain and compared with the sivasamy et al (2008) and it is also having good conformity also.

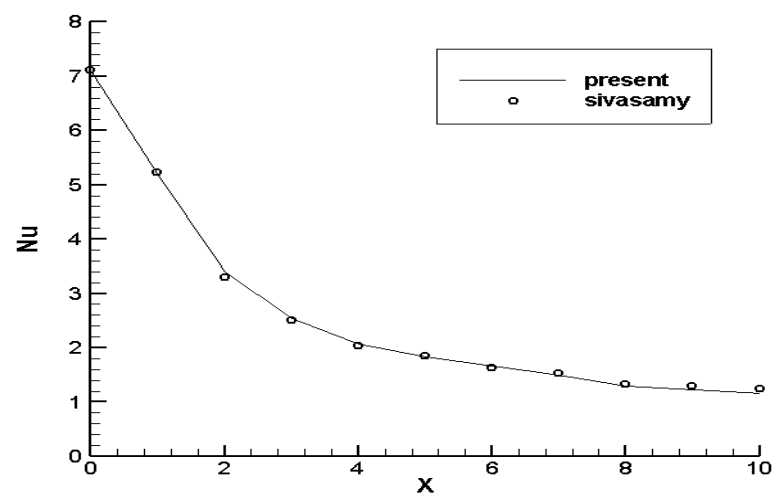

Fig 4.Validation of the bottom wall Nusselt number against Sivasamy et al [2008]

\subsection{Grid Independence Study}

Table 2 Grid Independence Study.

\begin{tabular}{|c|c|c|}
\cline { 3 - 3 } Grid level & $\begin{array}{c}\text { Total No } \\
\text { of nodes }\end{array}$ & $\begin{array}{c}\text { Average Nu Number for } \\
\text { the bottom wall }\end{array}$ \\
\hline \hline Grid 1 & 259246 & 8.31 \\
\hline Grid 2 & 274584 & 10.5 \\
\hline Grid 3 & 289584 & 11.367 \\
\hline Grid 4 & 304584 & 12.31 \\
\hline Grid 5 & 319922 & 12.3768 \\
\hline Grid 6 & 335260 & 12.4092 \\
\hline
\end{tabular}

Table 2 reveals the prediction of nusselt number for same aspect ratio and Reynolds number. From the table it is concluded that there was not a substantial change in Nusselt Number after Grid Number 4.So Grid Number 4 is considered as the grid selction for the particular aspect ratio.

\section{Numerical Approach and Procedures}

In the present numerical investigation the two dimensional laminar incompressible jet is considered for the present study. The single phase model is adopted in the present simulation for the sake of simplicity. No slip condition and no penetration conditions are applied for the velocity components on the solid wall. The present numerical study concentrates on heat transfer investigation in the presence of solid block with different shape of deflector. The algorithm used for solving is the semi implicit method for pressure linked equation algorithm. The numerical investigations based on finite volume method are simulated by ANSYS FLUENT 15.0 CFD code. The Tecplot 9.0 (postprocessor) is used to interpret the result, for obtaining the contour plots of $\mathrm{X}$ velocity, $\mathrm{Y}$ velocity and streamline contour for flow field.

\section{Results and Discussion}

The present author has already done heat transfer investigation in jet impingement on a solid block. Numerical simulations of heat transfer characteristics of 
jet impingement in the presence of baffles are presented. In the present investigation rectangular and semi circle (Dimple) shaped baffles are adopted. The influence of rectangular and dimple shaped baffles are presented interms of velocity of fluid and Nusselt number.

The following figures shows the temperature contour for different Reynolds number in the presence of dimple shaped baffles

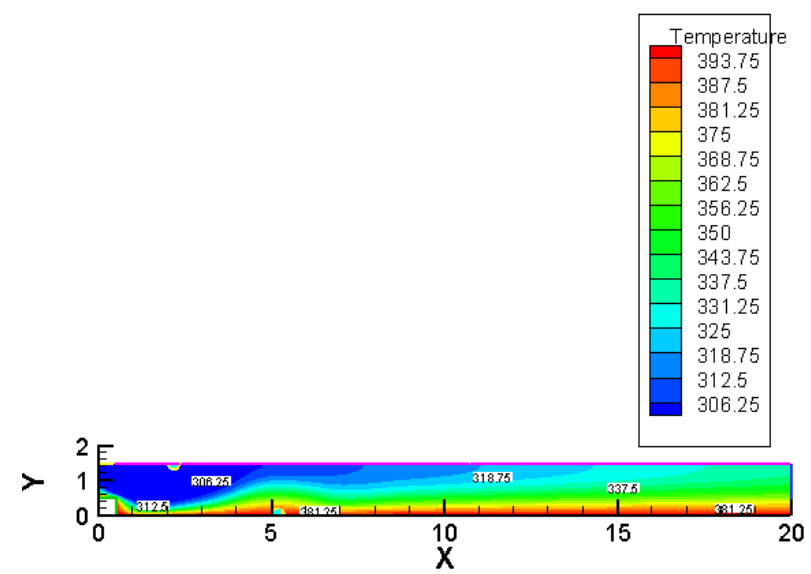

Fig. 5(a).Temperature contour in the presence of dimple baffle for $\mathrm{Re}=150$ for $\mathrm{AR}=3$

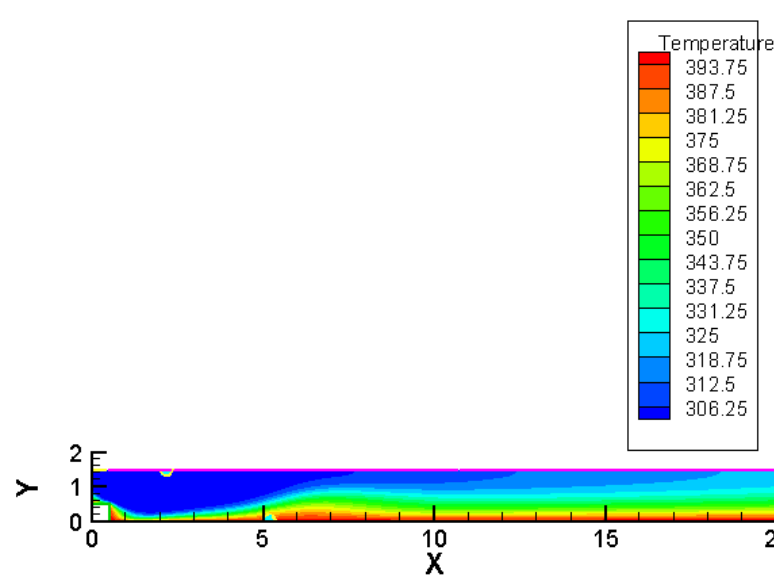

Fig. 5(b). Temperature contour in the presence of dimple baffle for $\mathrm{Re}=250$ for $\mathrm{AR}=3$

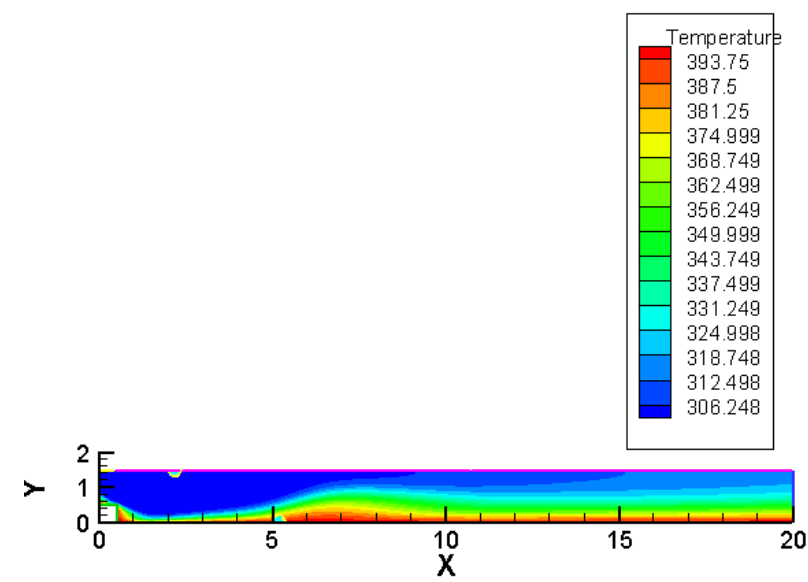

Fig. 5(c).Temperature contour in the presence of dimple baffle for $\mathrm{Re}=300$ for $\mathrm{AR}=3$
From figure 5 it is inferred that the average surface temperature increases with the increase of Reynolds number. When the Reynolds number increase the thermal

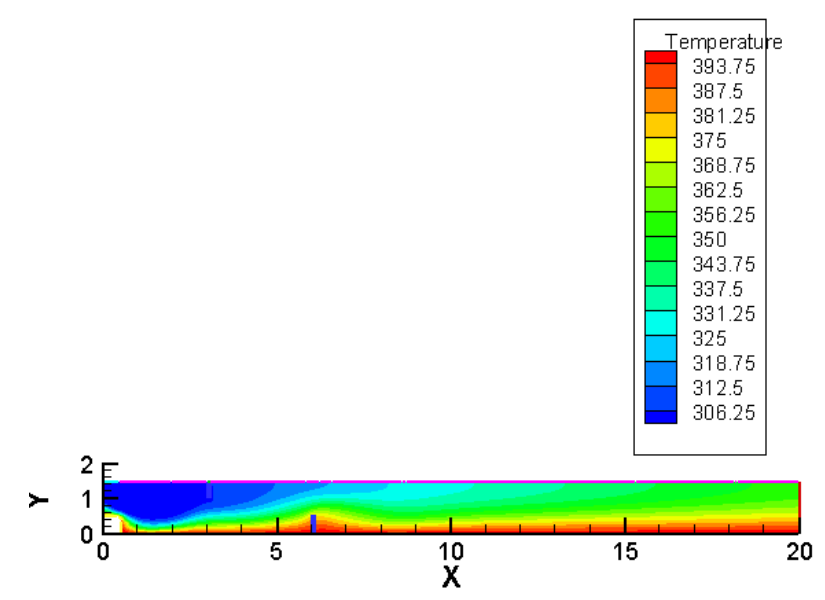

Fig 6(a).Temperature contour in the presence of rectangular baffle for $\mathrm{Re}=100$ for $\mathrm{AR}=3$

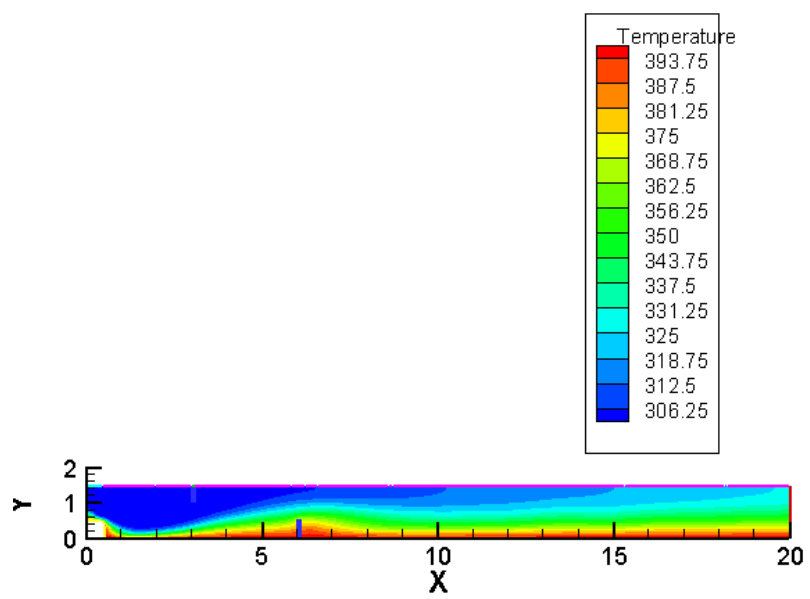

Fig. 6(b).Temperature contour in the presence of rectangular baffle for $\mathrm{Re}=200$ for $\mathrm{AR}=3$

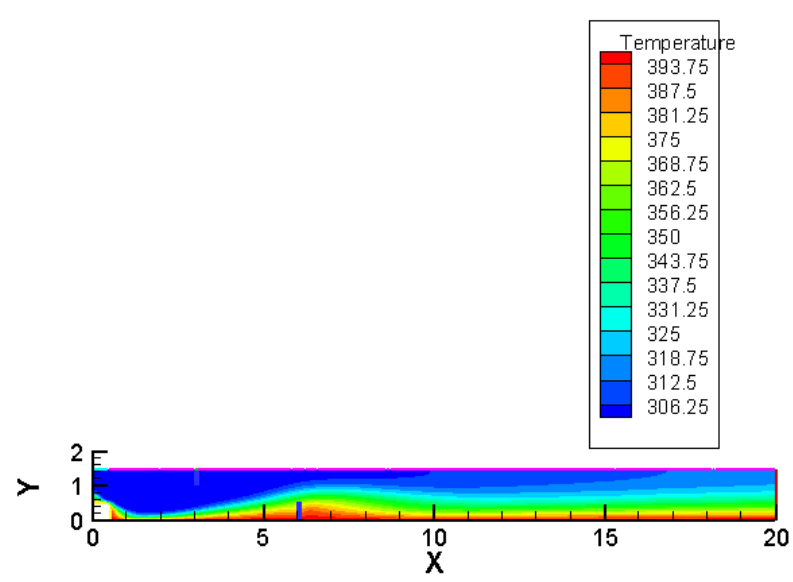

Fig.6(c).Temperature contour in the presence of rectangular baffle for $\mathrm{Re}=300$ for $\mathrm{AR}=3$ 
The figure 6 shows the temperature contour for different Reynolds number in the presence of rectangular baffles. From figure $5 \& 6$, it is concluded that the vortex induced by the baffle increases mixing and thus enhances the heat transfer. The figure $5 \& 6$ shows that there was a reduction in thickness of shear layer with the increase of Reynolds number.

The influence of baffle on the heat transfer can be determined by comparing the heat transfer characteristics downstream of the baffle with the presence of the baffle and without the presence of baffle. It is noticed that peak horizontal velocity in the presence of baffle is higher than peak horizontal velocity in the absence of baffle.

The followings table shows the peak Nusselt number with the presence of rectangular baffle $\&$ without baffle.

Table 3.Comparison of Peak Nusselt number between with and with out baffle in the computational domain.

\begin{tabular}{|l|l|l|}
\hline Re Number & $\begin{array}{l}\text { Peak Nu without } \\
\text { baffle }\end{array}$ & $\begin{array}{l}\text { Peak Nu with } \\
\text { baffle }\end{array}$ \\
\hline 100 & 9.881 & 11.4216 \\
\hline 200 & 14.856 & 16.2422 \\
\hline 300 & 20.4421 & 21.245 \\
\hline
\end{tabular}

Figure 7(a)\&7(b) Local Nusselt number in the downstream location in the presence of rectangular baffle.

For Reynolds number 100 nearly $15.59 \%$ improvement in Nusselt number and similary for $9.33 \%$ and $3.92 \%$ increment in for $\mathrm{Re}=200$ \& $\mathrm{Re}=300$ respectively.

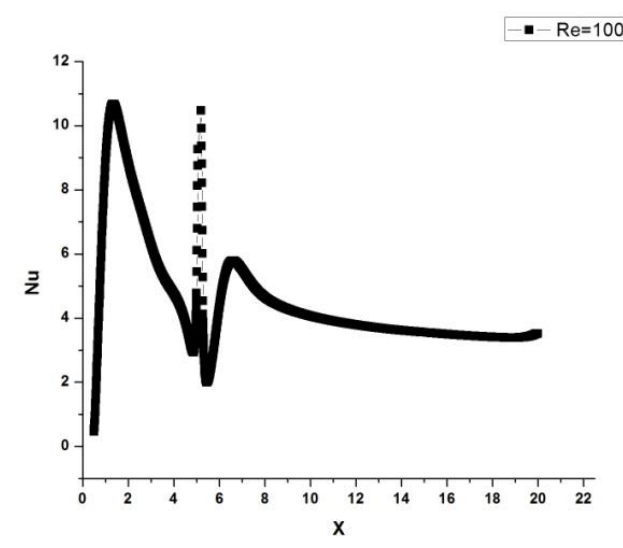

Fig. 7(a)

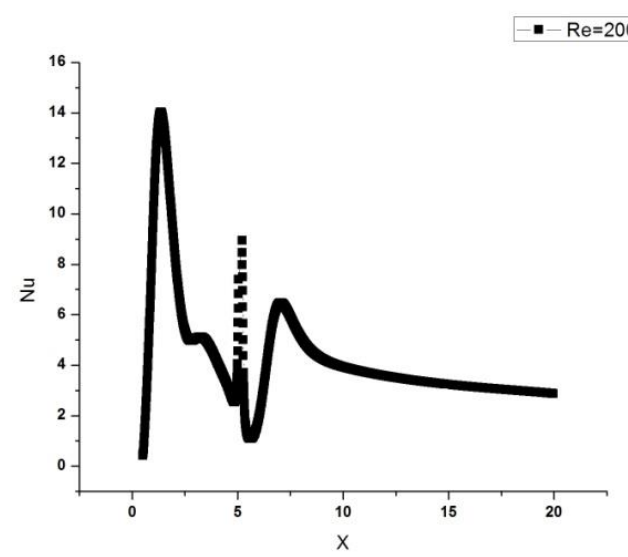

Fig. 7(b)
The figure 7(a) and 7(b)shows the local nusselt number in the downstream location.In the presence of rectangular baffle there are three peak nusselt number are identified in the downstream location. This will improve the average nussely number in the donstream location.Primary peak occurs at the reattachment of fluid in the computational domain. Seondary peak occurs due to the baffle and tertiary peak occurs due to high inertia force which cause flow sepeartion.

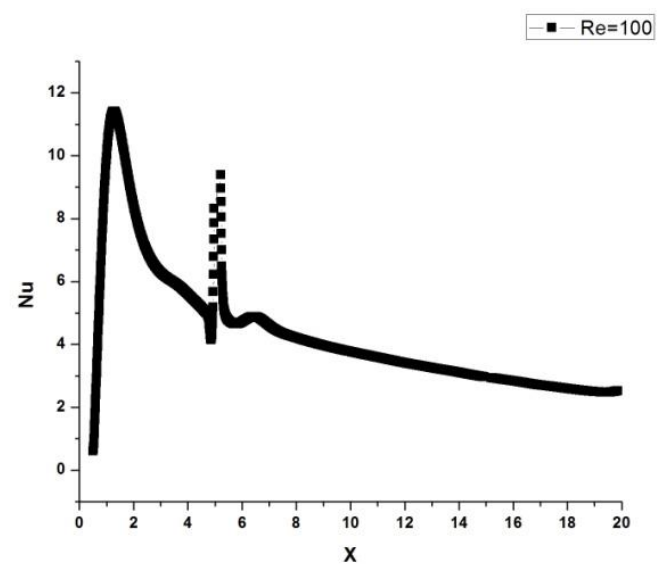

Fig 8(a)

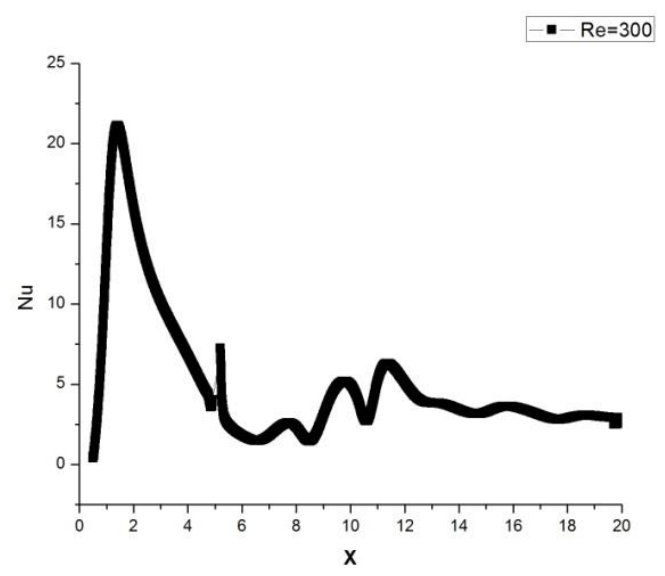

Fig 8(b)

Figure $8(a) \& 8(b)$ Local nusselt number in the downstream location in the presence of dimple baffle . In case of rectangular shaped baffles the form drag/resistance is more due to large pressure drop where as loss due to friction will be more in dimple shaped baffle. The overall loss is combination of these two.

Table 4.Peak Nusselt number for various shape deflectors

\begin{tabular}{|l|c|c|}
\hline \multirow{2}{*}{$\begin{array}{l}\text { Reynolds } \\
\text { Number }\end{array}$} & \multicolumn{2}{|c|}{ Peak Nusselt number } \\
\cline { 2 - 3 } & Rectangular & Dimple \\
\hline 100 & 7.02 & 8.16 \\
\hline 150 & 10.52 & 12.95 \\
\hline 200 & 13.46 & 14.02 \\
\hline 250 & 19.57 & 21.25 \\
\hline 300 & 23.70 & 23.58 \\
\hline
\end{tabular}

The table 4 shows that the presence of dimple shaped baffle produces high heat transfer compared to rectangular 
baffle.From the table 4 it is confirmed that the percentage of increase in nusselt number decreases with the increase of Reynolds number.

\section{Conclusion}

In the present numerical analysi of heat transfer, while the jet impinges on a block, is investigated using commercial CFD codes. The computed thermal fields in terms of the Nusselt number ranging from 2 to 5 with the Reynolds number ranging from 100 to 300 are analyzed in which the flow comes under the laminar regime.

Due to the presence of baffles, there was increase in nusselt number. The nusselt number profile along the downstream location is plotted for various Reynolds number.

The Nusselt number attains first peak value nearer to the reattachement length and second peak occurs nearer to the baffle and third peak occurs due to the higher Reynolds number

\section{References}

1. M.A. Habib, A.M. Mobarak, M.A. Sallak, E.A. Abdel Hadi, R.I. Affify, J Heat Trans-T ASME. 116, 363368 (1994)

2. Hussein M. Maghrabie,M. Attalla, H. E. Fawaz, M.Khalil, AEJ, 56, 3, 285-296 (2017)

3. WithadaJedsadaratanachai,NuthvipaJayranaiwachira, PongjetPromvonge, Chin. J. Chem. Eng. 23, 2, 342349 (2015)

4. Liu Haiyong,Liu Cunliang, Wu Wenming, ,CJA, 1616-1628 (2015).

5. S.Eiamsaard, S.Pattanapipat. Promvonge, JMST, 27, 7, 2199-2208 (2013)

6. Alsanossi M. Aboghrara', B.T.H.T. Baharudin, M.A. Alghoul, Nor Mariah Adam, A.A. Hairuddin, Husam A. Hasan, 10, 111-120 (2017).

7. Muthukannan.M, Rajeshkanna.P, Ankit Bajpai, Jeyakumar.S, AJSE, 39, 8077-8098 (2016),

8. Muthukannan.M, Rajeshkanna.P, Ankit Bajpai, Jeyakumar.S, JAFM, 9, 4, 1679-1694 (2016)

9. Sivasamy A, Selladurai V Rajeshkanna P, Int. J. Numer. Methods Fluids, 55, 965-983 (2007) 